\title{
Eficiência do uso da água pelo maracujazeiro amarelo sob diferentes níveis de irrigação e doses de potássio
}

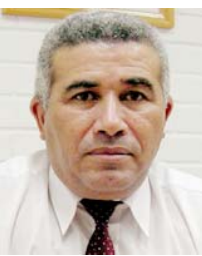

\author{
Valdemício F. de Sousa ${ }^{1}$, José A. Frizzone ${ }^{2}$, Marcos V. Folegatti² \& Thales V. de A. Viana ${ }^{3}$ \\ ${ }^{1}$ Embrapa Meio-Norte, Av. Duque de Caxias 5650, Bairro Buenos Aires, CP 01, CEP 64006-220, Teresina, PI. \\ Fone: (86) 225-1141. E-mail: vfsousa@cpamn.embrapa.br \\ ${ }^{2}$ ESALQ/USP, CEP 13418-900, Piracicaba, SP. Fone: (19) 3429-4217. E-mail: frizzone@carpa.ciagri.usp.br; \\ mvfolega@carpa.ciagri.usp.br \\ ${ }^{3}$ UFC, Campus do Pici -Bloco 804 Pici, CEP 60455-600, Fortaleza, CE. Fone: (85) 288-9754. E-mail: thales@ufc.com.br
}

Protocolo 77 - 6/5/2003 - Aprovado em 14/1/2005

\begin{abstract}
Resumo: Objetivou-se, com este trabalho, avaliar o efeito de quatro níveis de irrigação na eficiência do uso da água (EUA) pelo maracujazeiro amarelo, associados com doses de potássio aplicadas via fertirrigação por gotejamento. O experimento foi realizado na Fazenda Areão da ESALQ/USP, Piracicaba, SP $\left(22^{\circ} 42^{\prime} 30^{\prime \prime}\right.$ e $47^{\circ} 38^{\prime} 00^{\prime \prime}$ W). Os níveis de irrigação foram determinados com base no balanço médio de entrada e saída de água em quatro lisímetros (L), equivalentes a 0,25 ; 0,$50 ; 0,75$ e 1,00 de $\mathrm{L}$, respectivamente. As doses de $\mathrm{K}_{2} \mathrm{O}\left(\mathrm{kg} \mathrm{planta}^{-1} \mathrm{ano}^{-1}\right)$ foram: $\mathrm{O}\left(\mathrm{K}_{0}\right)$, $0,225\left(\mathrm{~K}_{1}\right), 0,450\left(\mathrm{~K}_{2}\right), 0,675\left(\mathrm{~K}_{3}\right)$ e 0,900 $\left(\mathrm{K}_{4}\right)$. A EUA foi determinada pela relação entre a produtividade comercial (PC) do maracujazeiro e os diferentes níveis de água (V). Os maiores $\left(34,05 \mathrm{~kg} \mathrm{ha}^{-1} \mathrm{~L}^{-1}\right)$ e os menores $\left(7,85 \mathrm{~kg} \mathrm{ha}^{-1} \mathrm{~L}^{-1}\right)$ valores de EUA pelo maracujazeiro, foram obtidos com os respectivos menores e maiores volumes de água aplicados. $\mathrm{O}$ aumento da água aplicada no maracujazeiro reduziu a EUA, enquanto o simples aumento da produtividade em função da quantidade de água aplicada não otimizou o recurso água. A EUA pelo maracujazeiro amarelo aumenta com a elevação das doses de potássio aplicadas via fertirrigação.
\end{abstract}

Palavras-chave: Passiflora edulis, manejo de fertirrigação, irrigação localizada

\section{Water use efficiency of the yellow passion fruit under differents irrigation levels and potassium doses}

\begin{abstract}
The objective of this work was to evaluate the effects of four irrigation levels on the water use efficiency (WUE) of the yellow passion fruit (Passiflora edulis Sims. f. flavicarpa Deg.), combined with different potassium doses applied by drip fertirrigation. The experiment was conducted at Fazenda Areão of ESALQ/USP, Piracicaba, São Paulo, Brazil $\left(22^{\circ} 42^{\prime} 30^{\prime \prime S}\right.$ and $\left.47^{\circ} 38^{\prime} 00^{\prime \prime} \mathrm{W}\right)$. The irrigation levels were established with average water consumption in the four lysimeters (L), equivalent to $0.25,0.50,0.75$ e 1.00 of $L$, respectively. The $\mathrm{K}_{2} \mathrm{O}$ doses $\left(\mathrm{kg} \mathrm{plant}^{-1}\right.$ year $\left.^{-1}\right)$ were: $0\left(\mathrm{~K}_{0}\right), 0.225\left(\mathrm{~K}_{1}\right), 0.450\left(\mathrm{~K}_{2}\right), 0.675\left(\mathrm{~K}_{3}\right)$ e $0.900\left(\mathrm{~K}_{4}\right)$. The WUE was determinated by relation between the of commercial yield (CY) of passion fruit for different potassium doses and water levels (V). The highest (34.05 $\mathrm{kg} \mathrm{ha}^{-1} \mathrm{~L}^{-1}$ ) and lowest (7.85 $\mathrm{kg} \mathrm{ha}^{-1} \mathrm{~L}^{-1}$ ) values of WUE by passion fruit were verified in the respective lower and higher water volume applied. The increase of water applied in passion fruit reduced the WUE, and the simple increase of yield as a function of amount of water applied did not optimized the water. The WUE by yellow passion fruit increased with the elevation of potassium doses applied through fertirrigation.
\end{abstract}

Key words: Passiflora edulis, fertirrigation management, drip irrigation

\section{INTRODUÇÃO}

A irrigação é indispensável para o maracujazeiro (Passiflora edulis Sims. f. flavicarpa Deg.), pois promove ótimo desenvolvimento das plantas, aumenta a produtividade, permite a obtenção de produção de forma contínua e uniforme, com frutos de boa qualidade. A falta de umidade no solo provoca a queda das folhas e dos frutos, principalmente no início de seu 
desenvolvimento e quando se formam, podendo crescer enrugados, prejudicando a qualidade da produção (Manica, 1981; Ruggiero et al., 1996).

A produtividade do maracujazeiro é muito afetada pela radiação solar, temperatura, número de horas de brilho solar e perda de umidade no solo. Fatores como estresse hídrico e deficiências nutricionais, associados a dias curtos e baixas temperaturas do ar e do solo restringem o crescimento e o potencial produtivo do maracujazeiro (Simon \& Karnatz, 1983; Menzel et al., 1986).

O fornecimento freqüente de água permite a floração e frutificação do maracujazeiro continuamente; no entanto, para a obtenção de boas produtividades com qualidade de frutos, a água deve ser aplicada em quantidade adequada. Carvalho et al. (2000) constataram que irrigações com lâminas de água superior a $75 \%$ da evaporação do tanque classe $\mathrm{A}$, resultaram na diminuição da massa média dos frutos e do rendimento do maracujazeiro amarelo.

A utilização adequada da água em cultivos irrigados tem condicionado aos produtores a garantia da produção; todavia, a maximização dos recursos naturais e o aumento da produtividade não se dão apenas com o fornecimento de água às culturas. A elevação dos níveis de fertilidade do meio exerce também destacada importância para o desenvolvimento e produção das plantas do maracujazeiro (Sousa et al., 2002).

$\mathrm{O}$ efeito da adubação mineral na produtividade do maracujazeiro é relatado por vários autores (Müller et al., 1979; Colauto et al., 1986; Martins, 1998; Carvalho et al., 2000); no entanto, a literatura ainda é limitada em artigos que tratam dos efeitos da combinação de fertilizantes e água de irrigação, principalmente em regime de fertirrigação.

O potássio é o elemento de maior mobilidade na planta e passa com facilidade de uma célula para outra e do xilema para o floema. Por isso, é o componente mineral de maior expressão nos processos osmóticos das plantas que envolvem absorção e armazenamento de água (Ruggiero et al., 1996).

A deficiência de potássio provoca redução na ramificação, no comprimento e nos diâmetros dos ramos, atraso na floração, redução no tamanho dos frutos e na área foliar do maracujazeiro, prejudicando a fotossíntese e o conteúdo de sólidos solúveis nos frutos (Ruggiero et al., 1996; Quaggio \& Piza Jr., 1998).

A eficiência do uso da água (EUA) relaciona a acumulação de biomassa ou produção comercial com a quantidade de água aplicada ou evapotranspirada pela cultura (Sousa et al., 2000). Em agricultura irrigada a elevação dos níveis de produção e a determinação da EUA é bastante complexa e requer conhecimentos interdisciplinares. Neste sentido, Dinar (1993) menciona os meios para se elevar os valores de EUA, destacando-se o manejo adequado de irrigação.

Dentre os meios e as técnicas adotadas para aumentar a eficiência do uso da água em agricultura irrigada, o emprego da irrigação por gotejamento com o fornecimento de água com alta freqüência e em baixo volume, tem-se mostrado adequado na elevação da EUA (Srinivas et al., 1989). Esses autores constataram que a máxima EUA pela cultura da melancia foi obtida com irrigação por gotejamento, quando as quantidades de água foram aplicadas com base em $25 \%$ da evaporação do tanque classe $\mathrm{A}$, devido ao pequeno estresse imposto e ao baixo decréscimo na produtividade, quando comparada com a alta redução no uso da água. Resultados semelhantes foram obtidos por Lin et al. (1983) que verificaram alta EUA sob baixo regime de irrigação em tomateiro.

Quando a eficiência do uso da água é determinada a partir da quantidade de água aplicada, Dinar (1993) e Letey (1993) destacam sua redução, porém sem diminuição da produção, como forma de aumentar a EUA. Neste aspecto, a escolha do sistema de irrigação (Dinar,1993) e redução do período de aplicação de água no ciclo da cultura (Richards et al., 1993 e Howell et al., 1998) constituem pontos significativos.

Ritshel et al. (1994) elevaram a eficiência de uso de água pelo meloeiro irrigado por gotejamento, com a suspensão antecipada da irrigação, ou seja, aos 56 dias após o plantio. Avaliando a eficiência de uso da água para o maracujazeiro, Martins (1998) constatou resposta linear decrescente, cujos valores de EUA variaram de 35,87 a 19,64 $\mathrm{kg} \mathrm{ha}^{-1} \mathrm{~mm}^{-1}$, para as lâminas totais de irrigação de $826 \mathrm{~mm}$ e $1.724 \mathrm{~mm}$, respectivamente.

A distribuição da água e a manutenção de níveis ótimos de umidade no solo durante todo o ciclo da cultura, reduzem as perdas de água por drenagem e os períodos de estresse hídrico da cultura, o que aumenta a EUA. Isto pode ser atingido com aplicações de água com maior freqüência e em pequenas quantidades (Lin et al, 1983; Srinivas et al., 1989; Mishra et al., 1995; Saeed \& El-Nadi, 1997; Sousa et al., 1998).

Este trabalho teve como objetivo avaliar os efeitos de quatro níveis de irrigação na eficiência do uso da água pelo maracujazeiro amarelo, associados com doses de potássio aplicadas via fertirrigação por gotejamento.

\section{MATERIAL E MÉTODOS}

O experimento foi realizado no Campo Experimental da Fazenda Areão, pertencente ao Departamento de Engenharia Rural da Escola Superior de Agricultura Luiz de Queiroz, ESALQ/USP, localizado no município de Piracicaba, São Paulo, na latitude de $22^{\circ} 42^{\prime} 30^{\prime \prime} \mathrm{S}$, longitude $47^{\circ} 38^{\prime} 00^{\prime \prime}$ e altitude 576 $\mathrm{m}$. Para a caracterização física e química do solo foram retiradas amostras nas camadas de $0-0,20 \mathrm{~m}, 0,20-0,40 \mathrm{~m}$ e $0,40-0,60 \mathrm{~m}$ (Tabelas 1 e 2).

Tabela 1. Caracterização química do solo da área experimental

\begin{tabular}{|c|c|c|c|c|c|c|c|c|c|}
\hline $\begin{array}{c}\text { Camada } \\
\text { (m) }\end{array}$ & $\begin{array}{c}\mathrm{pH} \\
\left(\mathrm{CaCl}_{2}\right)\end{array}$ & 10 & &  & & & & $\begin{array}{l}\mathrm{T} \\
(0\end{array}$ & $V$ \\
\hline 0-0, & 5, & 14 & & & & & &, 50 & 63 \\
\hline 0 & 5,0 & 11 & & & & & & 60 & 57 \\
\hline $0-0,60$ & 5,30 & 9 & & 0,50 & 32 & & & 0,50 & 64 \\
\hline
\end{tabular}

Tabela 2. Valores de densidade global, teores de argila, silte e areia do solo da área experimental

\begin{tabular}{|c|c|c|c|c|c|c|}
\hline \multirow{2}{*}{$\begin{array}{c}\text { Camada } \\
\text { M }\end{array}$} & $\mathrm{CC}$ & PMP & \multirow{2}{*}{$\begin{array}{c}\text { Densidade } \\
\mathrm{kg} \mathrm{dm}^{-3}\end{array}$} & Argila & Silte & Areia \\
\hline & $\mathrm{cm}^{3}$ & $\mathrm{~cm}^{-3}$ & & \multicolumn{3}{|c|}{$\mathrm{g} \mathrm{kg}^{-1}$} \\
\hline $0-0,20$ & 0,4148 & 0,3472 & 1,49 & 624,0 & 172,0 & 204,0 \\
\hline $0,20-0,40$ & 0,4193 & 0,3491 & 1,46 & 694,3 & 138,3 & 167,4 \\
\hline $0,40-0,60$ & 0,4500 & 0,3808 & 1,43 & 688,9 & 136,4 & 174,8 \\
\hline
\end{tabular}


O preparo do solo consistiu-se de duas gradagens com posterior aplicação e incorporação de $2,0 \mathrm{t} \mathrm{ha}^{-1}$ de calcário dolomítico 60 dias antes do transplantio das mudas, para elevação da saturação por base a $80 \%$.

As covas foram abertas no espaçamento de $3,5 \times 4,0 \mathrm{~m}$, formato cilíndrico com $1,0 \mathrm{~m}$ de diâmetro e $0,5 \mathrm{~m}$ de profundidade. A adubação de fundação foi feita incorporando-se, a cada, cova $40 \mathrm{~L}$ de esterco de curral, $0,20 \mathrm{~kg} \mathrm{P}_{2} \mathrm{O}_{5}$ (superfosfato simples), 0,004 kg de Zn (sulfato de zinco) e 0,001 kg de B (ácido bórico).

O sistema de irrigação utilizado foi por gotejamento, com emissores de vazão 2,4 e $4 \mathrm{~L} \mathrm{~h}^{-1}$. Os gotejadores foram distribuídos em quantidade variada por planta e dispostos em círculo, com raio médio de $0,40 \mathrm{~m}$ do caule da planta, de acordo com a lâmina aplicada em cada tratamento.

A quantidade de água aplicada foi definida com base no balanço médio de entrada e saída de água em lisímetros de drenagem, com superfície circular de $1,23 \mathrm{~m}^{2}$ e profundidade de $1,5 \mathrm{~m}$, instalados no centro da área experimental, considerandose a precipitação efetiva e a eficiência do sistema de irrigação. Para a determinação da lâmina de água equivalente ao máximo nível de irrigação, utilizaram-se as Eqs. 1 e 2.

$$
\begin{gathered}
V=\frac{n_{g} q T i}{E_{f}} \\
L=\frac{V}{A_{1}}
\end{gathered}
$$

em que:

V - volume de água aplicado em cada lisímetro, $\mathrm{L}$

$\mathrm{n}_{\mathrm{g}}$ - número de gotejadores no lisímetro

q - vazão do gotejador, $\mathrm{L} \mathrm{h}^{-1}$

Ti - tempo de irrigação;

$\mathrm{E}_{\mathrm{f}}$ - eficiência de irrigação, 0,90

$\mathrm{L}$ - lâmina máxima de irrigação, $\mathrm{mm}$

$\mathrm{A}_{1}$ - área do lisímetro, $\mathrm{m}^{2}$

O momento da irrigação foi definido em função do potencial matricial, entre - 8,5 e - 10,0 kPa, medido nos tensiômetros instalados nos lisímetros nas profundidades de 0,$10 ; 0,30 ; 0,50$ e $0,70 \mathrm{~m}$. O monitoramento da umidade no solo se deu indiretamente, por intermédio de tensiômetros instalados a 0,30 $\mathrm{m}$ do caule da planta e nas já mencionadas.

Utilizou-se o delineamento experimental de blocos ao acaso em parcela subdividida e quatro repetições. Os tratamentos se compunham de quatro níveis de irrigação (subparcelas) e cinco doses de potássio (parcelas). Os níveis de irrigação foram: $\mathrm{L}_{1}=$ $0,25 \mathrm{~V}(926,76 \mathrm{~L}) ; \mathrm{L}_{2}=0,50 \mathrm{~V}(1.528,20 \mathrm{~L}) ; \mathrm{L}_{3}=0,75 \mathrm{~V}(2.117,28 \mathrm{~L}) ;$ $\mathrm{L}_{4}=1,00 \mathrm{~V}(2.706,36 \mathrm{~L})$.

No período de maio a julho de 1999, o manejo de água foi realizado de tal forma a aplicar quantidade d'água uniforme para todos os tratamentos, para garantir o pegamento das mudas e o estabelecimento da cultura. A partir do mês de agosto as quantidades d'água foram diferenciadas de acordo com os tratamentos estabelecidos, resultando na aplicação dos volumes totais $926,73 \mathrm{~L}, 1.528,20 \mathrm{~L}, 2.117,28 \mathrm{Le} 2.706,36 \mathrm{~L}$ para os níveis de irrigação $\mathrm{L}_{1}, \mathrm{~L}_{2}, \mathrm{~L}_{3}$ e $\mathrm{L}_{4}$, respectivamente.
As adubações de formação e de produção com nitrogênio, foram definidas segundo as recomendações de Meletti \& Maia (1999) e feitas por meio da água de irrigação, na freqüência de aplicação de sete dias. Aplicaram-se, nas fases de formação e de produção, 0,10 e $0,38 \mathrm{~kg}$ de $\mathrm{N}$ por planta, respectivamente. As dosagens de $\mathrm{K}$ foram definidas para as duas fases, conforme os tratamentos estabelecidos (Tabela 3 ), sendo que na primeira fase se utilizou cloreto de potássio e, na segunda, nitrato de potássio.

$\mathrm{Na}$ fase de formação a distribuição dos nutrientes ao longo do ciclo da cultura foi feita de forma linear, em 15 aplicações. Durante a fase de produção a aplicação dos nutrientes foi feita em 32 vezes, conforme a marcha de absorção adaptada de Haag et al. (1973).

A eficiência do uso da água (EUA) foi determinada pela relação entre a produtividade comercial (PC) do maracujazeiro obtido com as doses de potássio: $\mathrm{K}_{0}, \mathrm{~K}_{1}, \mathrm{~K}_{2}, \mathrm{~K}_{3}$ e $\mathrm{K}_{4}$ e os diferentes volumes de água $(\mathrm{V})$ aplicados pela irrigação, Eq.3.

$$
\mathrm{EUA}=\frac{\mathrm{PC}}{\mathrm{V}}
$$

Tabela 3. Doses de potássio ( $\mathrm{kg}$ de $\mathrm{K}_{2} \mathrm{O}$ por planta), nas fases de formação e produção

\begin{tabular}{cccc}
\hline Níveis de potássio & Formação & Produção & Total \\
\hline $\mathrm{K}_{0}$ & 0 & 0 & 0 \\
$\mathrm{~K}_{1}$ & 0,025 & 0,200 & 0,225 \\
$\mathrm{~K}_{2}$ & 0,050 & 0,400 & 0,450 \\
$\mathrm{~K}_{3}$ & 0,075 & 0,600 & 0,675 \\
$\mathrm{~K}_{4}$ & 0,100 & 0,800 & 0,900 \\
\hline
\end{tabular}

\section{RESULTADOS E DISCUSSÃO}

Os maiores valores de produtividade comercial foram obtidos nos tratamentos: $\mathrm{L}_{3} \mathrm{~K}_{3}\left(44.540 \mathrm{~kg} \mathrm{ha}^{-1}\right), \mathrm{L}_{2} \mathrm{~K}_{2}(38.717 \mathrm{~kg}$ $\left.\mathrm{ha}^{-1}\right), \mathrm{L}_{3} \mathrm{~K}_{2}\left(35.613 \mathrm{~kg} \mathrm{ha}^{-1}\right) \mathrm{e} \mathrm{L}_{2} \mathrm{~K}_{3}\left(34.033 \mathrm{~kg} \mathrm{ha}^{-1}\right)$ (Tabela 4). Pelos resultados verifica-se que maiores rendimentos de frutos comerciais do maracujazeiro amarelo foram obtidos com níveis intermediários de irrigação (aplicação de 1.520,20 e 2.706,30 L planta $^{-1}$ ano $^{-1}$ ), tal como foi observado por Martins (1998) e Carvalho et al. (2000), que constataram efeitos quadráticos de lâminas de irrigação sobre a produtividade da cultura.

Obteve-se a eficiência do uso da água em função dos diferentes níveis de irrigação, empregando-se modelos

Tabela 4. Produtividade comercial do maracujazeiro sob

\begin{tabular}{|c|c|c|c|c|c|}
\hline \multirow{2}{*}{$\begin{array}{c}\text { Doses de } \\
\mathrm{K}_{2} \mathrm{O} \\
\left(\mathrm{kg} \mathrm{planta}^{-1}\right)\end{array}$} & \multicolumn{5}{|c|}{ Níveis de irrigação ( $\mathrm{L}_{\text {planta }}{ }^{-1}$ ano $^{-1}$ ) } \\
\hline & 926,76 & 1528,2 & 2110 & 2706,3 & Média \\
\hline 0 & 169 & 17364 & 18084 & 1 & 18 \\
\hline 0,225 & 214 & 244 & 254 & & 23 \\
\hline 0,450 & 22 & $38^{\circ}$ & 35 & 66 & 7,93 \\
\hline 0,675 & 607 & 3403 & & &, 62 \\
\hline 0,900 & & & & & 31035,20 \\
\hline Média & 23921,20 & 29153,22 & 31491,16 & 24433,18 & 27249,69 \\
\hline
\end{tabular}
diferentes níveis de irrigação e doses de potássio, aplicadas por fertirrigação 
A.

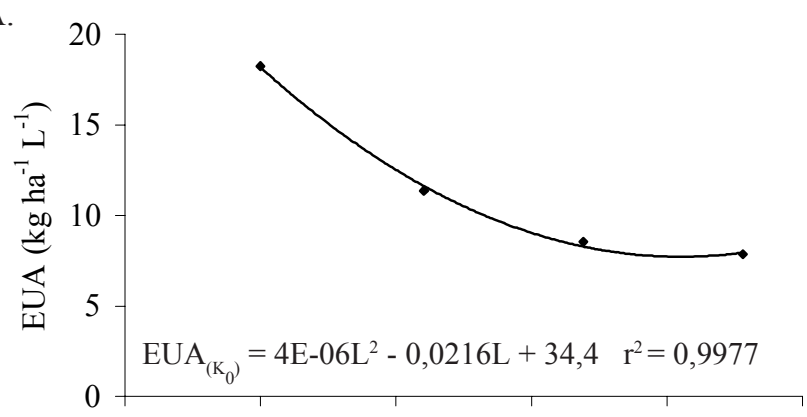

B.

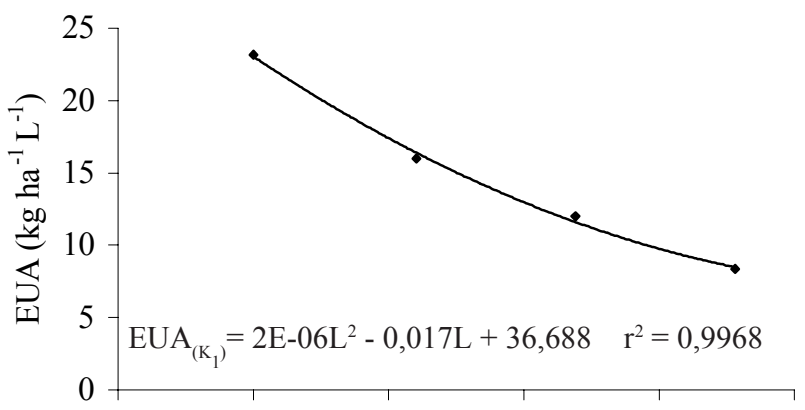

C.

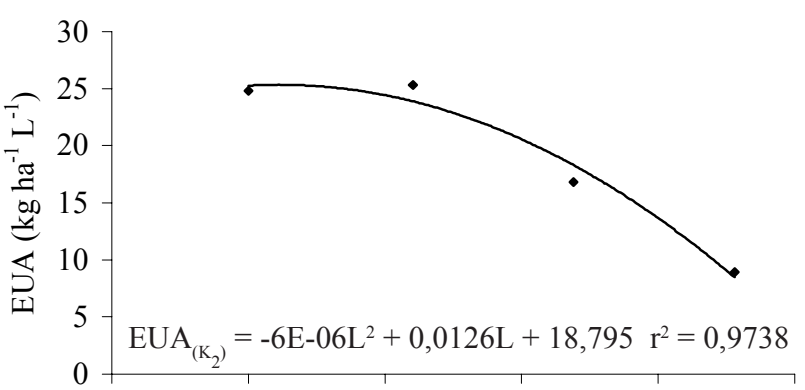

D.

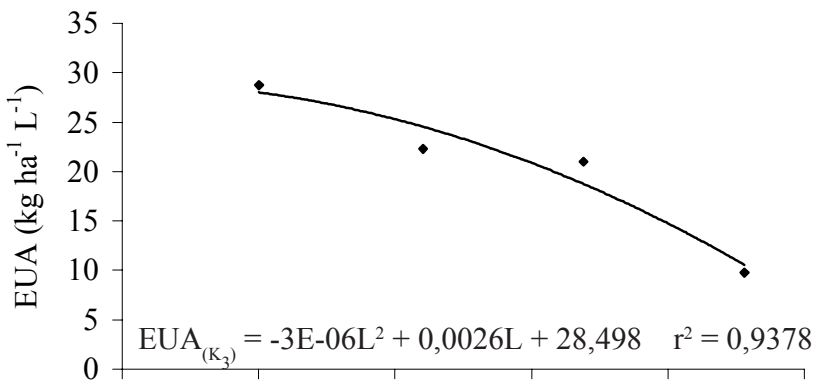

E.

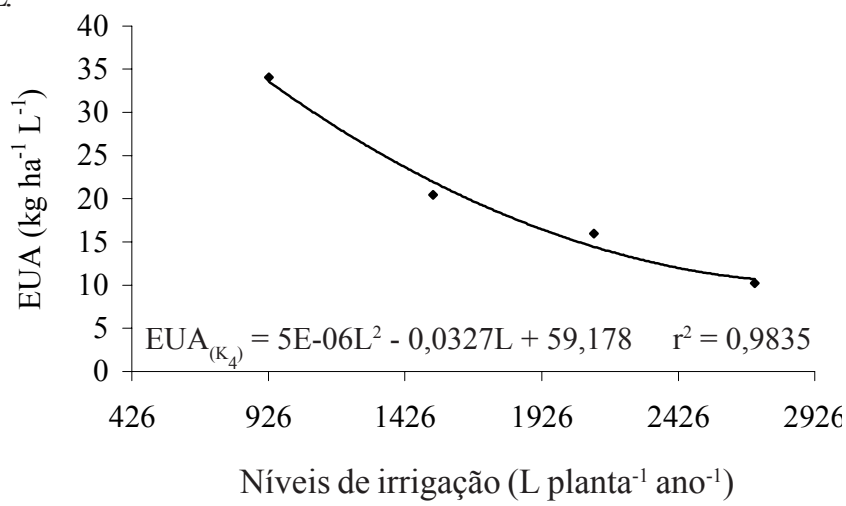

Figura 1. Níveis de irrigação e doses de potássio $\mathrm{K}_{0}-0$ (A), $\mathrm{K}_{1}$ $-0,225$ (B), $\mathrm{K}_{2}-0,450$ (C), $\mathrm{K}_{3}-0,675$ (D) e $\mathrm{K}_{4}-0,90 \mathrm{~kg} \mathrm{planta}^{-1}$ (E) aplicada por fertirrigação na eficiência do uso da água (EUA) pelo maracujazeiro amarelo polinomiais quadráticos, cujos coeficientes de determinação, $r^{2}=0,9977, r^{2}=0,9968, r^{2}=0,9738, r^{2}=0,9378$ e r $^{2}=0,9835$, nas doses de potássio $\mathrm{K}_{0}, \mathrm{~K}_{1}, \mathrm{~K}_{2}, \mathrm{~K}_{3}$ e $\mathrm{K}_{4}$, respectivamente, revelaram ajustes adequados entre níveis de irrigação e EUA (Figura 1A, B, C, D e E). O comportamento dos dados nas respectivas figuras diverge de Martins (1998), que constatou efeito linear de lâminas de água sobre a eficiência do uso da água pelo maracujazeiro amarelo.

Fazendo-se uma análise geral do comportamento da eficiência do uso da água em função dos níveis de irrigação constata-se decréscimo acentuado da EUA com o aumento da quantidade de água aplicada pela irrigação, exceto em $\mathrm{K}_{2}$, em que foi observado um pequeno acréscimo na EUA do primeiro para o segundo nível de água aplicado (Figura 1C), o que se atribui ao incremento da produtividade no segundo nível de irrigação $\left(\mathrm{L}_{2}\right)$ que não se deu na mesma proporção da água aplicada, como ocorreu nos demais casos, indicando que os maiores e os menores valores de eficiência do uso da água pelo maracujazeiro foram obtidos com os respectivos menores e maiores volumes de água aplicados e, em parte, evidencia que o aumento do volume de água aplicada no maracujazeiro reduz a eficiência do uso da água. Evidencia, também, que o simples aumento da produtividade em função da quantidade de água aplicada nem sempre otimiza o recurso água; este fenômeno está coerente com as conclusões apresentadas por Letey (1993), Ritshel et al. (1994) e Sousa et al. (2000), ao mencionarem que, na maioria das vezes, o aumento da EUA pode ser obtido devido ao decréscimo da quantidade de água aplicada.

No que se refere às doses de potássio, os menores e maiores valores de eficiência do uso da água pelo maracujazeiro, dentro de cada tratamento com K, (18,25 e 7,85 $\mathrm{kg} \mathrm{ha}^{-1} \mathrm{~L}^{-1}$ ) (Figura 1A) e $\left(34,05\right.$ e 10,19 $\left.\mathrm{kg} \mathrm{ha}^{-1} \mathrm{~L}^{-1}\right)$ (Figura 1E), foram obtidos nas combinações dos níveis de irrigação com $\mathrm{K}_{0}$ e $\mathrm{K}_{4}$, respectivamente. O incremento de produtividade do maracujazeiro pode ser atribuída, em maior parte, aos efeitos da adubação potássica, uma vez que se registrou tendência no aumento da EUA, com aplicação de dosagens de potássio. Esses pontos extremos observados para EUA, foram diferentes daqueles obtidos por Martins (1998), com variação de 35,87 a $19,64 \mathrm{~kg} \mathrm{ha}^{-1} \mathrm{~mm}^{-1}$, no maracujazeiro amarelo, o que pode ser atribuído às diferenças entre produtividades e as quantidades de água, uma vez que este autor considerou as precipitações pluviométricas e, além disso, a própria unidade $(\mathrm{mm})$ utilizada para expressar a água aplicada, enquanto neste trabalho se utilizou a unidade de volume (L).

\section{CONCLUSÕES}

1. A eficiência do uso da água pelo maracujazeiro amarelo foi reduzida com o aumento do volume de água aplicado.

2. A eficiência do uso da água pelo maracujazeiro amarelo aumenta com a elevação das dosagens de potássio aplicadas via fertirrigação. 


\section{LITERATURA CITADA}

Carvalho, A.J.C. de; Martins, D.P.; Monnerat, P.H.; Bernardo, S. Adubação nitrogenada e irrigação no maracujazeiroamarelo. I. Produtividade e qualidade dos frutos. Pesquisa Agropecuária Brasileira, Brasília, v.35, n.6, p.1101-1108, 2000.

Colauto, N.M.; Manica, I.; Reboldi, J.; Mielniczuk, J. Efeito do nitrogênio, fósforo e potássio sobre a produção, qualidade e estado nutricional do maracujazeiro amarelo. Pesquisa Agropecuária Brasileira, Brasília, v.21, n.7, p.691-695, 1986.

Dinar, A. Economic factors and opportunities as determinantes of water use efficiency in agriculture. Irrigation Science, Heidelberg, v.14, p.47-52, 1993.

Haag, H.P.; Oliveira, G.D.; Borduchi, A.S.; Sarruge, J.R. Absorção de nutrientes por duas variedades de maracujá. ESALQ/ USP. Anais... Escola Superior de Agricultura "Luiz de Queiroz” Universidade de São Paulo, v. 30, p. 267-279, 1973.

Howell, T.A.; Tolk, J.A.; Schneider, A.D.; Evett, S.R. Evapotranspiration, yield, and water use efficiency of corn hybrids differing in maturity. Agronomy Journal, Madison, v.90, p.3-9, 1998.

Letey, J. Relationship between salinity and efficient water use. Irrigation Science, Heidelberg, v.14, p.75-84, 1993.

Lin, S.S.M.; Hubbel, J.N.; Samson Isou, S.C.S.; Splittstoesser, W.E. Drip irrigation and tomato yield under tropical conditions. Hortscience, Fort Collins, v.18, p.460-161, 1983.

Manica, I. Fruticultura tropical: Maracujá. São Paulo: Agronômica Ceres, 1981.151p.

Martins, D.P. Resposta do maracujazeiro amarelo (Passiflora edulis Sins var. favicarpa Deg.) a lâminas de irrigação e doses de nitrogênio e potássio. Campos dos Goytacazes: Centro de Ciências e Tecnologias da Universidade Estadual do Norte Fluminense, 1998. 84p. Tese Doutorado

Meletti, L.M.M.; Maia, M.L. Maracujá: produção e comercialização. Campinas: Instituto Agronômico de Campinas, 1999. 26p. Boletim Técnico, 181

Menzel, C.M.; Simpson, D.R.; Dowling, A.J. Water relations in passion fruit: Effect of moisture stress on growth, flowering and nutrient uptake. Scientia Horticulturae, Amsterdam. v.29, p.239-349, 1986.

Mishra, H.S.; Rathore, T.R.; Tomar, V.S. Water use efficiency of irrigated wheat in the Tarai Region of India. Irrigation Science, Heidelberg, v.16, p.75-80, 1995.

Müller, C.H.; Pinheiro, R.V.R.; Casali, V.W.D.; Oliveira, L.M. de; Manica, I.; Sousa, A.C.G. de. Efeitos de doses de sulfato de amônio e de cloreto de potássio sobre a produtividade e a qualidade de maracujás colhidos em épocas diferentes. Revista Ceres, Viçosa, v.26, n.143, p.48-64, 1979.
Quaggio, J.A.; Pizza Jr., C. de T. Nutrição mineral da cultura do maracujá. In: Ruggiero, C. In: Simpósio Brasileiro Sobre a Cultura do Maracujá, 5, Jaboticabal, 1998, Anais... Jaboticabal: FUNEP, 1998, p.130-156.

Richards, R.A.; López-Castañeda, C.; Gomez-Macpherson, H.; Condon. A.G. Improving the effiency of water use by plant breedingand molecular biology. Irrigation Science, Heidelberg, v.14, p.93-104, 1993.

Ritschel, P.S.; Sousa, V.F. de; Conceição, M.A.F.; Souza, V.A.B. de; Coêlho, E.F. Efeito da época de suspensão da irrigação na produtividade do meloeiro (Cucumis melo L.). In: Congresso Brasileiro de Irrigação e Drenagem, 10., 1994, Salvador. Anais ... Salvador: ABID, 1994. p.135-142.

Ruggiero, C.; São José, A.R.; Volpe, C.A., Oliveira, J.C. de, Durigan, J.F.; Buamgartner, J.G.; Silva, J.R. da; Nakamura, K.; Ferreira, M.E.; Kavati, R.; Pereira, V. de P. Maracujá para exportação: aspectos técnicos da produção. Brasília: EMBRAPA-SPI, 1996, 64p. Publicações Técnicas FRUPEX, 19

Saeed, I.A.M.; El-Nadi, A.H. Irrigation effects on the growth, yield, and water use efficiency of alfalfa. Irrigation Science, Heidelberg, v.17, p.63-68, 1997.

Simon, P.; Karnatz, A. Effect of soil and air temperature on growth and flower formation of purple passion fruit (Passiflora edulis Sins). Acta Horticulturae, Wageningen, v.139, p.120-128, 1983.

Sousa, V.F. de; Coêlho, E.F.; Fizzone, J.A.; Folegatti, M.V.; Andrade Júnior, A.S.; Oliveira, F. das C. Freqüência de irrigação por gotejamento na eficiência do uso da água no meloeiro. In: Congresso Brasileiro de Engenharia Agrícola, 27., 1998, Poços de Caldas. Anais.... Poços de Caldas: SBEA, 1998. p.214-216.

Sousa, V.F. de; Coêlho, E.F.; Fizzone, J.A.; Folegatti, M.V.; Andrade Júnior, A.S.; Oliveira, F. das C. Eficiência do uso da água pelo meloeiro sob diferentes freqüências de irrigação. Revista Brasileira de Engenharia Agrícola e Ambiental, Campina Grande, v.4, n.2, p.183-188, 2000.

Sousa, V.F. de; Folegatti, M.V.; Coelho Filho, M.A.; Fizzone, J.A. Distribuição radicular do maracujazeiro sob diferentes doses de potássio aplicadas por fertirrigação. Revista Brasileira de Engenharia Agrícola e Ambiental, Campina Grande, v.6, n.1, p.51-56, 2002.

Srinivas, K.; Hegede, D.M.; Havanagi, G.V. Plant water relations, canopy temperature, yield and water-use efficiency of water melon (Citrulls lanatus (Thamb.) Matsum et Nakai) under drip and furrow. Journal of Horticultural Science, Ashford, v.64,n.1, p.115-124, 1989. 